\title{
Long Noncoding RNA SOX2-OT Knockdown Inhibits Proliferation and Metastasis of Prostate Cancer Cells Through Modulating the miR-452-5p/HMGB3 Axis and Inactivating Wnt $/ \beta$-Catenin Pathway
}

\author{
Xiaofei Song,, Hang Wang, ${ }^{2}$ Jiawen Wu, ${ }^{1}$ and Yang Sun ${ }^{1}$
}

\begin{abstract}
Background: Recent studies have proven that abnormal expression of long noncoding RNAs (lncRNAs) often contributes to growth and invasion of cancer cells. The purpose of this study was to investigate the biological function and regulatory mechanism of lncRNA SOX2 overlapping transcript (SOX2-OT) in prostate cancer (PCa) progression.

Materials and Methods: The expression of SOX2-OT, microRNA-452-5p (miR-452-5p), and high mobility group box 3 (HMGB3) was detected by quantitative real-time polymerase chain reaction (qRT-PCR). Flow cytometry was performed to determine the cell cycle distribution. Western blot assay was conducted to measure the protein levels of cyclin D1, p21, p27, E-cadherin, vimentin, and $N$-cadherin. The interaction between miR452-5p and SOX2-OT or HMGB3 was predicted by bioinformatics analysis and verified by dual-luciferase reporter assay and RNA immunoprecipitation assay. The mice xenograft model was established to investigate the role of SOX2-OT in vivo.

Results: SOX2-OT and HMGB3 were upregulated, whereas miR-452-5p was downregulated in PCa tissues and cells. Knockdown of SOX2-OT inhibited PCa cell growth and metastasis. MiR-452-5p could directly bind to SOX2-OT and its knockdown reversed the inhibitory effects of SOX2-OT interference on growth and metastasis of PCa cells. HMGB3 was a direct target of miR-452-5p and its knockdown weakened the promotive effects of miR-452-5p silence on growth and metastasis of PCa cells. Moreover, HMGB3 expression was inversely regulated by miR-452-5p and positively modulated by SOX2-OT. Furthermore, SOX2-OT activated the Wnt/ $\beta$-catenin signaling pathway through increasing HMGB3 expression. Finally, SOX2-OT knockdown hindered tumor growth in vivo by regulating miR-452-5p/HMGB3 axis.

Conclusions: SOX2-OT downregulation limited PCa cell growth and metastasis by regulating miR-452-5p/ HMGB3 axis and inactivating Wnt/ $\beta$-catenin signaling pathway, which might offer lncRNA-directed diagnosis and therapy for PCa.
\end{abstract}

Keywords: HMGB3, miR-452-5p, prostate cancer, SOX2-OT, Wnt/ $\beta$-catenin signaling pathway

\section{Introduction}

$\mathbf{P}$ rostate cancer $(\mathrm{PCa})$ is the second most common cancer among men and is the fifth leading cause of cancer death in the world. ${ }^{1}$ Although treatment of PCa has made significant progress over the past few decades, such as chemo- therapy, radiotherapy, and immunotherapy, the 5-year survival rate for PCa patients is $<30 \%$. $^{2,3}$ Besides, effective and specific strategies to reduce the incidence and mortality of PCa are still lacking. Thus, it is necessary to elucidate the pathogenesis of $\mathrm{PCa}$ and develop novel therapies for clinical application.

${ }^{1}$ Department of Urology, Fudan University, Minhang Hospital, Shanghai, China.

${ }^{2}$ Department of Urology, Fudan University, Zhongshan Hospital, Shanghai, China. 
Long noncoding RNAs (lncRNAs) are a class of long RNAs that are $>200$ nucleotides in length without translation capacity, and have been confirmed to be involved in numerous types of cancer development, including PCa. ${ }^{4,5}$ For example, lncRNA SChLAP1 facilitated PCa cell proliferation and metastasis through sponging miR-198 and promoting the MAPK1 pathway. ${ }^{6}$ Moreover, lncRNA suppressed the progression of PCa by regulating miR-9-5p/ QKI-5 axis. ${ }^{7}$ LncRNA SOX2 overlapping transcript (SOX2OT) is aberrantly expressed in multiple diseases, including human lung cancer, ${ }^{8}$ esophageal squamous cell carcinoma, ${ }^{9}$ breast cancer, ${ }^{10}$ and PCa. ${ }^{11}$ Nevertheless, the specific effect and molecule mechanism of SOX2-OT in PCa remain to be fully elucidated.

The competitive endogenous RNA (ceRNA) hypothesis has been proposed in a variety of cancers, suggesting that lncRNA can serve as microRNA (miRNA) sponges to regulate the expression of related genes. ${ }^{12}$ miRNAs, a class of short noncoding RNAs, affect gene expression by suppressing messenger RNA (mRNA) translation or mediating mRNA degradation. ${ }^{13}$ It has been confirmed that microRNA-452-5p (miR-452-5p) is lowly expressed in PCa tissues and its aberrant expression has a pivotal role in the tumorigenesis of $\mathrm{PCa} .{ }^{14}$ However, up to now, there is no relevant report about the interaction of SOX2-OT and miR452-5p in PCa.

High mobility group box 3 (HMGB3; also known as HMG2a,) belongs to HMGB family. ${ }^{15}$ HMGB3 has been suggested to participate in cell growth, metastasis, and apoptosis of various cancer cells. ${ }^{16,17}$ Previous research has proven that HMGB3 is expressed at a high level in PCa tissues and significantly related to patients' shorter diseasefree survival. ${ }^{18}$ According to the bioinformatics software, there are possible binding sites between miR-452-5p and SOX2-OT or HMGB3. Thus, we assumed that SOX2-OT might act as a molecular sponge for miR-452-5p to modulate HMGB3 expression.

In our research, the expression of SOX2-OT, miR-452$5 \mathrm{p}$, and HMGB3 was measured in PCa tissues and cells. Moreover, we explored their effects on cell growth and metastasis and investigated the ceRNA regulatory network of SOX2-OT/miR-452-5p/HMGB3. This research aimed to offer new insights into understanding the molecular mechanisms of SOX2-OT in PCa.

\section{Materials and Methods}

\section{Clinical samples and cell culture}

In this study, 27 pairs of PCa tissues and adjacent normal samples were obtained from PCa patients undergoing surgery at Minhang Hospital, Fudan University. These patients did not receive any treatment before surgery. Each patient provided written informed consent before tissue collection. The study was permitted by Research Ethics Committee of Minhang Hospital, Fudan University. Excised fresh tissues were put in liquid nitrogen at once and then kept at $-80^{\circ} \mathrm{C}$ for the following experiments.

Normal human prostate epithelial cell line (NPrEC) and $\mathrm{PCa}$ cell lines (LNCaP and DU145) were bought from BeNa Culture Collection (Beijing, China). Dulbecco's modified Eagle's medium (DMEM; Invitrogen, Waltham, MA) containing $10 \%$ fetal bovine serum (FBS; Invitrogen) was used for culturing cells. The cells were incubated in a moist incubator with $\mathrm{CO}_{2}(5 \%)$ at $37^{\circ} \mathrm{C}$.

\section{Cell transfection}

The small-interfering RNA against SOX2-OT or HMGB3 (si-SOX2-OT or si-HMGB3) and their negative control (si$\mathrm{NC}$ ), miR-452-5p mimic or inhibitor (miR-452-5p or antimiR-452-5p) and corresponding negative control (miR-NC or anti-miR-NC), SOX2-OT overexpression plasmid (pcDNA-SOX2-OT) and its negative control (pcDNA-NC) were bought from GenePharma (Shanghai, China). Lentivirus-mediated shRNA interference targeting SOX2OT (sh-SOX2-OT) and its negative control (sh-NC) were constructed by Genechem (Shanghai, China). The sequences used for cell transduction were listed in Supplementary Table S1. LNCaP and DU145 cells were transfected with these vectors using Lipofectamine 3000 (Invitrogen).

\section{Quantitative real-time polymerase chain reaction}

TRIzol reagent (Invitrogen) was used for RNA extraction. The complementary DNA (cDNA) for SOX2-OT, HMGB3, and miR-452-5p were synthesized by TaqMan Reverse Transcription Kit and TaqMan microRNA Reverse Transcription Kit (Applied Biosystems, Foster City, CA). Subsequently, quantitative real-time polymerase chain reaction (qRT-PCR) was performed using the SYBR Green Master Mix (Vazyme, Nanjing, China) on ABI7500 system (Applied Biosystems).

In this study, the primers were listed as follows: SOX2-OT (forward, 5'-GTTCATGGCCTGGACTCTCC-3'; reverse, 5'ATTGCTAGCCCTCACACCTC-3'); miR-452-5p (forward, 5'-AGCGCGAACTGTTTGCAGAGGA-3'; reverse, 5'-ATCC AGTGCAGGGTCCGAGG-3'); HMGB3 (forward, 5'-ATTC GGAATTCCGTATCTGGCCTTTTGAC-3'; reverse, 5'-CGG TTACTCGGCTTACGCTTGGACTG-3'); $\beta$-actin (forward, 5'-CTGGAACGGTGAAGGTGAC-3'; reverse, 5'-CGGCCA CATTGTGAACTTTG-3'); and U6 (forward, 5'-ACCCTGAG AAATACCCTCACAT-3'; reverse, 5'-GACGACTGAGCCC CTGATG-3'). The expression of SOX2-OT, HMGB3, and miR-452-5p was evaluated with the $2^{-\Delta \Delta \mathrm{Ct}}$ method and normalized with $\beta$-actin (for SOX2-OT and HMGB3) or U6 (for $\operatorname{miR}-452-5 \mathrm{p})$.

\section{Cell cycle assay}

The cells were collected after transfection for $48 \mathrm{~h}$, and fixed using ice-cold ethanol $\left(70 \%,-20^{\circ} \mathrm{C}, 24 \mathrm{~h}\right)$. Then, the cells were centrifuged and washed using phosphate-buffered saline (PBS) and then hatched using propidium iodide (PI) solution $(25 \mu \mathrm{g} / \mathrm{mL})$ in PBS containing Triton X-100 $(0.2 \%)$ and RNase $(50 \mu \mathrm{g} / \mathrm{mL})$ for $30 \mathrm{~min}$ at $37^{\circ} \mathrm{C}$. Finally, the cell cycle distribution was analyzed using flow cytometry (Guava Technologies, Hayward, CA).

\section{Western blot assay}

RNA immunoprecipitation assay (RIPA) lysis buffer (Sangon Biotech, Shanghai, China) was used to gain total protein. Then, bicinchoninic acid protein assay kit (Tanon, Shanghai, China) was used to determine the protein concentration. The protein $(\sim 40 \mu \mathrm{g})$ was resolved by sodium dodecyl sulfate-polyacrylamide gel electrophoresis and then 
transferred onto the polyvinylidene fluoride (PVDF) (Beyotime) membranes.

Five percent nonfat dried milk (Yili, Beijing, China) was used to block the membranes. Then the membranes were probed with primary antibody against cyclin D1 (1:2000, ab226977; Abcam, Cambridge, United Kingdom), p21 (1:1000, ab227443; Abcam), p27 (1:1000, ab215434; Abcam), E-cadherin (1:500, ab15148; Abcam), vimentin (1:2000, ab137321; Abcam), $N$-cadherin (1:1000, ab76057; Abcam), HMGB3 (1:1000, D260490; Sangon Biotech), or $\beta$-actin $\left(1: 2000\right.$, ab8227; Abcam) at $4^{\circ} \mathrm{C}$ for $12-16 \mathrm{~h}$. Afterward, all membranes were maintained in horseradish peroxidase (HRP)-conjugated anti-rabbit immunoglobulin $\mathrm{G}$ (IgG; 1:4000, D110058; Sangon Biotech). Finally, all protein bands were observed with the ECL reagent (Tanon) and qualified using ImageJ software.

\section{Transwell assay}

Twenty-four-well transwell chamber (Corning, MA) was used to evaluate cell migration and invasion. In brief, LNCaP and DU145 cells (in $200 \mu \mathrm{L}$ serum free medium) were placed on the upper chambers pretreated with Matrigel (Becton Dickinson) (without Matrigel treatment for migration assay). Medium containing 10\% FBS $(600 \mu \mathrm{L})$ was placed in the bottom chambers. After $24 \mathrm{~h}$ incubation, cells that had migrated and invaded through the pores were fixed by methanol and stained with crystal violet $(0.1 \%)$. Finally, migrated and invaded cells were observed and counted using a microscope.

\section{Dual-luciferase reporter assay}

The putative binding sites of miR-452-5p and SOX2-OT or HMGB3 were predicted by starBase v2.0 (http://starbase .sysu.edu.cn/) or TargetScan (www.targetscan.org). SOX2OT or HMGB3 $3^{\prime}$ untranslated region (3'UTR) fragment including putative or mutated binding sites of miR-452-5p were synthesized and then cloned into a pmirGLO reporter vector (Promega, Madison, WI), namely wild-type plasmids (WT-SOX2-OT, WT-HMGB3) or mutated-type plasmids (MUT-SOX2-OT, MUT-HMGB3). These plasmids were cotransfected with miR-452-5p (or miR-NC) into LNCaP and DU145 cells. Dual-luciferase reporter assay system (Promega) was performed to measure the luciferase activity after transfection for $48 \mathrm{~h}$, followed by normalizing to Renilla luciferase activity.

\section{RIP assay}

Magna RIP Kit (Millipore, Billerica, MA) was used for RIP assay. In brief, LNCaP and DU145 cells were lysed by RIP lysis buffer, and then incubated using RIP buffer containing magnetic beads conjugated with anti-argonaute 2 (anti-Ago2) or IgG antibodies. Afterward, Proteinase $\mathrm{K}$ buffer was utilized to digest the protein, followed by RNA purification. Finally, qRT-PCR analysis was used to estimate the enrichment of SOX2-OT or HMGB3.

\section{Xenograft tumor model}

Male nude mice (5 weeks old) were provided by Shanghai Experimental Animal Center (Shanghai, China). The mice were randomly divided into two groups: sh-NC group $(n=6)$ and sh-SOX2-OT group $(n=6)$. Subsequently, the mice models were established by subcutaneously injecting DU145 cells stably transfected with sh-NC or sh-SOX2-OT. From the 1st week, tumor volume for each mouse was examined every week using a caliper and calculated using the formula: volume $=$ length $\times$ width $^{2} \times 0.5$. These mice were killed after 4 weeks, and tumor tissues were excised, weighed, and collected for experiments. This animal experiment was approved by Animal Care and Use Committee of Minhang Hospital, Fudan University.

\section{Statistical analysis}

GraphPad Prism 5.0 software (San Diego, CA) was used for statistical analysis. All data were presented as mean \pm standard deviation from at least three independent experiments. The correlation between miR-452-5p and SOX2-OT or HMGB3 was analyzed by Pearson's correlation. Kaplan-Meier method was used to generate survival curve. The significance of differences between two groups or multiple groups was assessed using Student's $t$-test or one-way analysis of variance (ANOVA). $p<0.05$ was regarded as statistically significant.

\section{Results}

\section{SOX2-OT level was enhanced in PCa tissues and cells}

First, qRT-PCR was conducted to measure the abundance of SOX2-OT in PCa tissues. SOX2-OT level was elevated in $\mathrm{PCa}$ tissues relative to adjacent normal tissues (Fig. 1A). Similarly, SOX2-OT level was also enhanced in PCa cells (LNCaP and DU145) in comparison with normal prostate epithelial cell line (NPrEC) (Fig. 1B). The low SOX2-OT expression group $(n=15)$ and high SOX2-OT expression group $(n=12)$ were classified in accordance with the mean value of SOX2-OT expression level in $\mathrm{PCa}$ patients. We observed that overall survival was lower in the high SOX2-OT expression group than in the low SOX2-OT expression group (Fig. 1C).

In addition, the relationship between the relative expression of SOX2-OT and clinical characteristics in patients with PCa was analyzed. As given in Supplementary Table S2, high expression level of SOX2-OT was significantly associated with tumor size, serosal invasion, lymph metastasis, and tumor-node-metastasis (TNM) stages, but not with gender and age. These findings showed that SOX2OT expression was enhanced in $\mathrm{PCa}$ and was reversely associated with overall survival.

\section{Knockdown of SOX2-OT inhibited PCa cell proliferation and metastasis}

To test the biological role of SOX2-OT in PCa progression, loss-of-function experiments were performed through transfection of si-SOX2-OT. QRT-PCR was used to examine the transfection efficiency. Results uncovered that the transfection of si-SOX2-OT markedly declined the expression SOX2-OT (Fig. 2A), implying that a favorable efficiency of SOX2-OT knockdown was obtained. Flow cytometry was used to assess cell cycle distribution. Results presented that the ratio of G0/G1 phase cells was evidently 

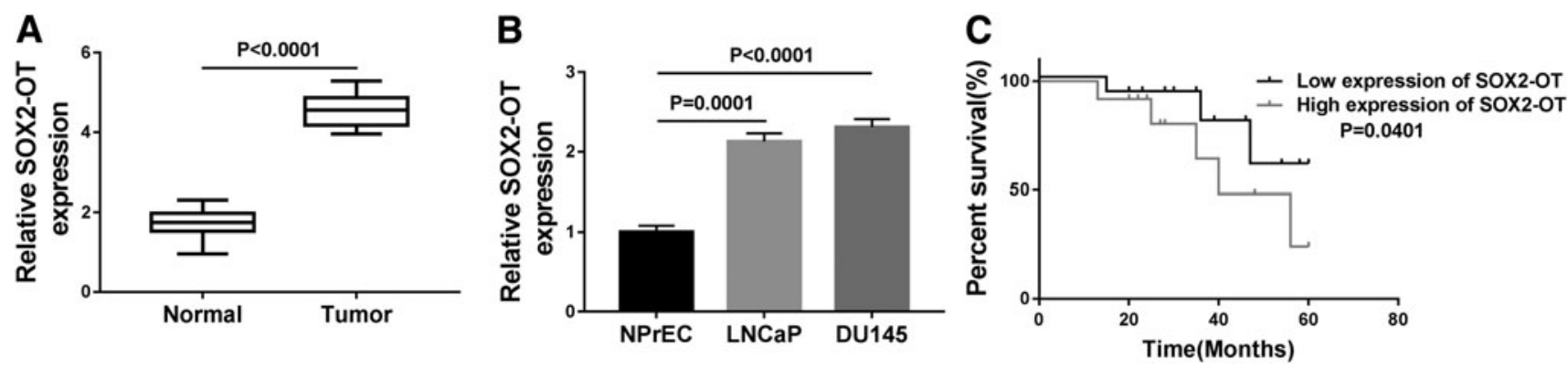

FIG. 1. SOX2-OT expression was enhanced in PCa tissues and cells. (A) SOX2-OT level was measured by qRT-PCR in PCa tissues and adjacent normal tissues. (B) The abundance of SOX2-OT was detected using qRT-PCR in PCa cells (LNCaP and DU145) and NPrEC. (C) The survival rate was analyzed between low and high SOX2-OT expression groups in PCa patients. NPrEC, normal human prostate epithelial cell line; PCa, prostate cancer; qRT-PCR, quantitative real-time polymerase chain reaction; SOX2-OT, SOX2 overlapping transcript.

elevated after SOX2-OT knockdown, whereas the ratio of S and $\mathrm{G} 2 / \mathrm{M}$ phase cells was obviously reduced after interference of SOX2-OT (Fig. 2B), suggesting that knockdown of SOX2-OT could block the cell cycle progression at the G0/G1 phase to suppress cell growth.

Next, we further investigated the cell cycle/growth-related proteins by Western blot assay. It is widely reported that both p21 and p27 (belonging to the kinase inhibitor protein family) serve as cyclin-dependent kinase (CDK) inhibitors and inhibit the growth of different cell types, and cyclin D1 (belonging to a family of three closely associated D-type cyclins) is required for cell cycle progression in G1 and is a growth-promoting protein. ${ }^{19,20}$ Western blot assay demonstrated that deficiency of SOX2-OT reduced the protein abundance of cyclin D1, and promoted the expression of p21 and p27 (Fig. 2C). Moreover, silencing SOX2-OT repressed LNCaP and DU145 cell migration and invasion (Fig. 2D, E).

It is widely acknowledged that epithelial-mesenchymal transition (EMT) is a critical step toward metastasis of cancer. ${ }^{21}$ Hence, EMT-related proteins were measured in PCa (LNCaP and DU145) cells after transfection with siSOX2-OT. As given in Figure 2F, E-cadherin (an epithelial marker) expression was remarkably elevated and the expression of vimentin and $\mathrm{N}$-cadherin (mesenchymal markers) was drastically decreased after transfection with siSOX2-OT. Taken together, our findings disclosed that SOX2-OT interference blocked cell cycle in the G0/G1 phase and inhibited metastasis of PCa cells.

\section{MiR-452-5p was a target of SOX2-OT in PCa cells}

As lncRNAs exert their biological function by binding with their downstream miRNAs [26], we predicted the potential targets of SOX2-OT using the starbase tool. As given in Figure 3A, miR-452-5p was predicted as a target of SOX2-OT. To confirm whether SOX2-OT directly targeted miR-452-5p, the dual-luciferase reporter and RIP assays were carried out. Results presented that transfection of miR-452-5p greatly lowered the luciferase activity of WT-SOX2-OT in LNCaP and DU145 cells, whereas no clear change of MUT-SOX2-OT luciferase activity was detected in LNCaP and DU145 cells after transfection with miR-452-5p or miR-NC (Fig. 3B). In addition, RIP assay revealed that SOX2-OT enrichment was apparently increased in miR-452-5p group coated with Ago2 antibody with respect to the control group (Fig. 3C).
Next, we explored the influence of SOX2-OT on the level of miR-452-5p. As given in Figure 3D, miR-452-5p level was elevated in cells transfected with si-SOX2-OT in contrast to those cells transfected with si-NC. Moreover, the miR-452-5p abundance was reduced in $\mathrm{PCa}$ tissues and cells relative to their corresponding controls (Fig. 3E, F). Besides, the correlation between miR-452-5p and SOX2-OT was analyzed in PCa tissues. Results disclosed that miR-452-5p abundance was inversely associated with SOX2-OT level in PCa tissues $(r=-0.7232, p<0.0001)$ (Fig. 3G). Based on all the above, we demonstrated that miR-452-5p directly interacted with SOX2OT and was negatively regulated by SOX2-OT.

\section{Deficiency of miR-452-5p could reverse the suppressive effects of SOX2-OT interference on growth and metastasis of PCa cells}

To investigate whether SOX2-OT-mediated PCa progression was modulated by miR-452-5p, rescue experiments were performed by cotransfection of si-SOX2-OT and anti-miR452-5p. First, the transfection efficiency of anti-miR-452-5p was determined using the qRT-PCR analysis. Results displayed that miR-452-5p expression was strikingly decreased in LNCaP and DU145 cells after transfection with miR-452-5p (Fig. 4A), suggesting that transfection of anti-miR-452-5p was successful.

In addition, miR-452-5p knockdown lowered the ratio of cells in G0/G1 phase, whereas it promoted the ratio of cells in $\mathrm{S}$ and G2/M phases (Fig. 4B). Moreover, interference of miR$452-5 p$ could reverse the impact of SOX2-OT downregulation on increase in G0/G1 phase cells and decrease in S and G2/M phases cells (Fig. 4B). Likewise, inhibition of miR-452-5p led to an obvious increase in cyclin D1 expression and decrease in p21 and p27 expression, and miR-452-5p silence weakened the impacts of SOX2-OT deficiency on inhibition of cyclin D1 expression and promotion of p21 and p27 expression in LNCaP and DU145 cells (Fig. 4C, D).

Furthermore, we observed that transfection of anti-miR452-5p facilitated LNCaP and DU145 cell migration and invasion, and partially abolished the anti-migration and antiinvasion impacts caused by SOX2-OT knockdown (Fig. 4E, F). Besides, we also found that miR-452-5p knockdown reduced $E$-cadherin abundance and elevated vimentin and $\mathrm{N}$-cadherin expression, and attenuated the effects of siSOX2-OT on promotion of $E$-cadherin expression and 


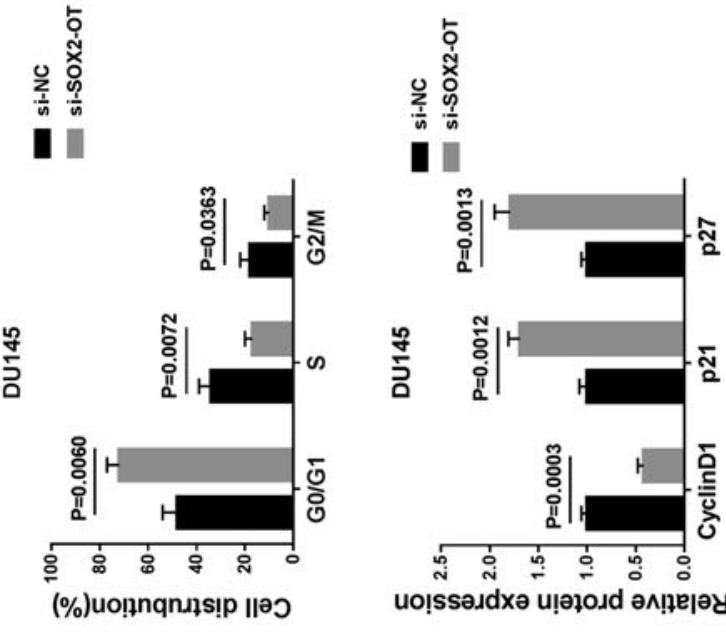

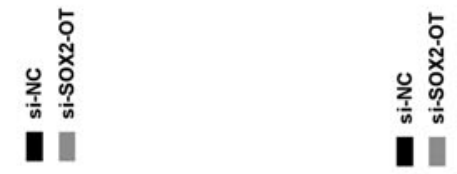

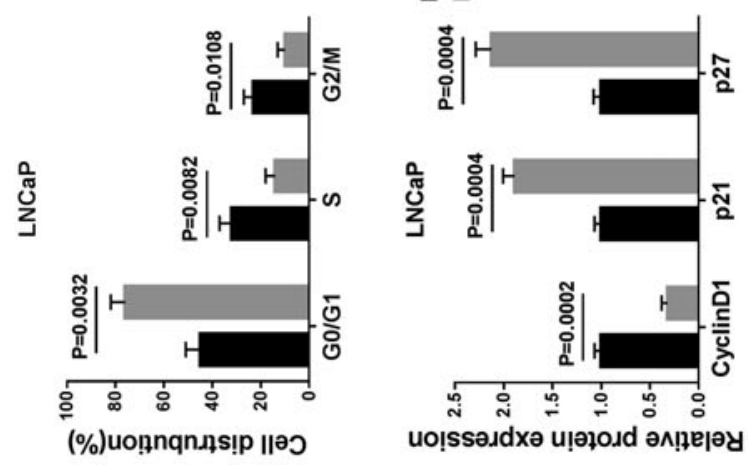

$\mathbf{m}$

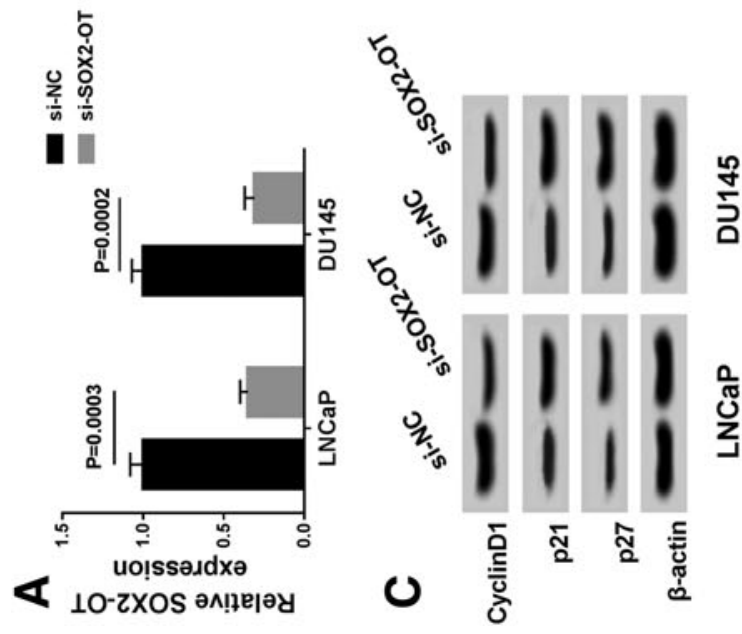

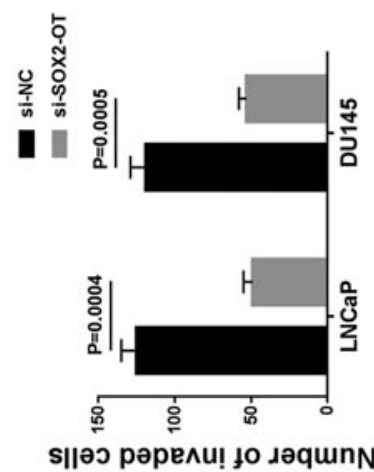

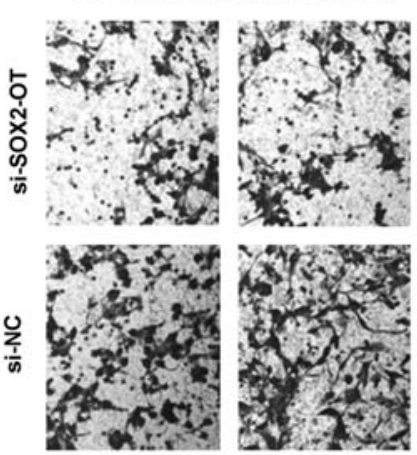

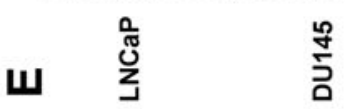

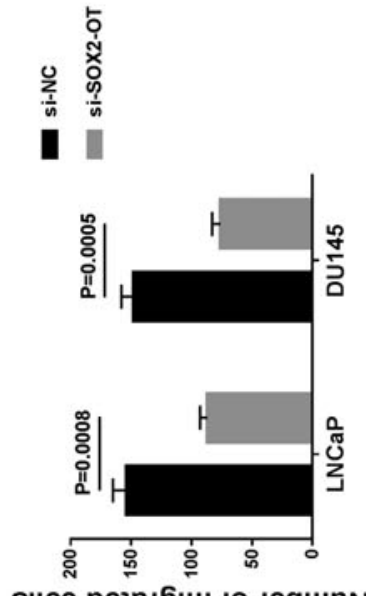

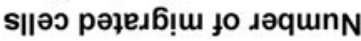

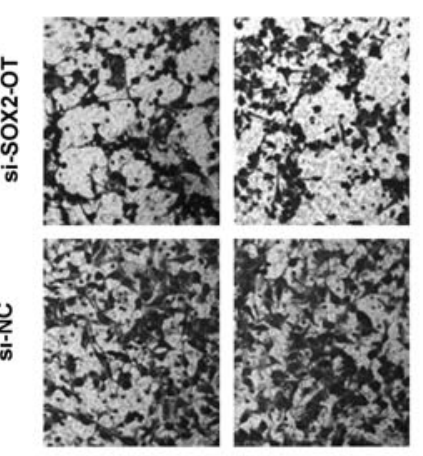

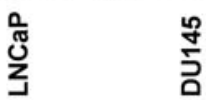

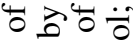

둥 뭉

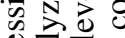

育

\&

20워

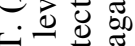

ธิ

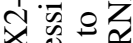

응

它 0

흥ㅎㅎ

U 0

两西学

들 त

उัง

$\Xi \overrightarrow{0} \cdot \frac{1}{n}$

过司

记

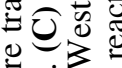

苍国.

0

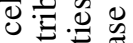

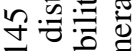

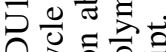

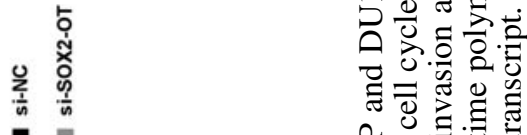

1.

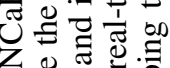

. ㄷ

它

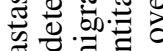

ษ。

.

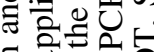

픙

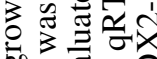

कo 3 त्र

ฮั

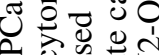

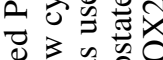

응일

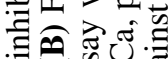

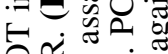

원효

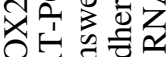

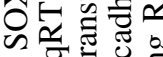

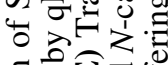

흐요월

.

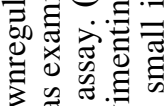

登。

느을

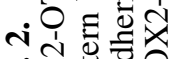
نx 

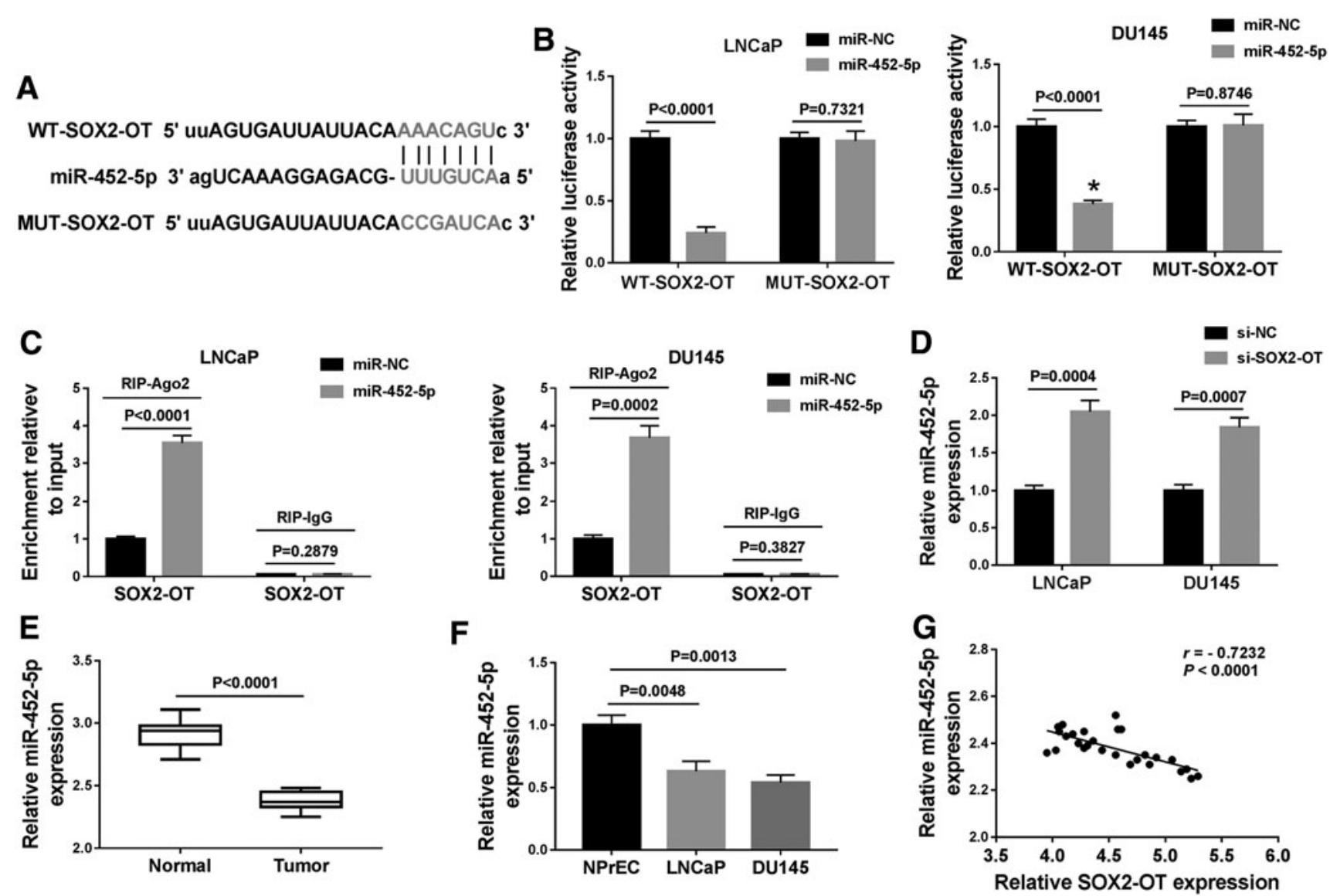

FIG. 3. SOX2-OT directly interacted with miR-452-5p in PCa cells. (A) Predicted binding sites between miR-452-5p and SOX2-OT were shown. (B) After cotransfection with miR-452-5p or miR-NC and WT-SOX2-OT or MUT-SOX2-OT, dualluciferase reporter assays were used to examine luciferase activity in LNCaP and DU145 cells. (C) RIP assay was performed to estimate SOX2-OT enrichment level in LNCaP and DU145 cells after transfection with miR-452-5p or miR-NC. (D) The abundance of miR-452-5p was assessed using qRT-PCR in LNCaP and DU145 cells transfected with si-NC or si-SOX2-OT. (E), F) The abundance of miR-452-5p was analyzed by qRT-PCR in PCa tissues, adjacent normal tissues, PCa cells (LNCaP and DU145), and NPrEC. (G) The correlation between SOX2-OT level and miR-452-5p expression was analyzed in PCa tissues. miR-452-5p, microRNA-452-5p; NPrEC, normal human prostate epithelial cell line; PCa, prostate cancer; qRT-PCR, quantitative real-time polymerase chain reaction; RIP, RNA immunoprecipitation; si-NC, small interfering RNA against negative control; si-SOX2-OT, small interfering RNA against SOX2-OT; SOX2-OT, SOX2 overlapping transcript.

inhibition of vimentin and $N$-cadherin expression (Fig. 4G, $\mathrm{H})$. All these results demonstrated that SOX2-OT knockdown repressed PCa cell growth and metastasis by upregulating miR-452-5p.

\section{HMGB3 was a downstream target of miR-452-5p in PCa cells}

To investigate the mechanism of action of miR-452-5p, we predicted the targets of miR-452-5p. TargetScan showed that HMGB3 contained potential binding sites of miR-4525p (Fig. 5A). We performed luciferase reporter and RIP assays to determine whether miR-452-5p interacted with HMGB3. As given in Figure 5B, upregulation of miR-4525 p significantly suppressed WT-HMGB3 luciferase activity, whereas MUT-HMGB3 luciferase activity was not evidently affected after upregulating miR-452-5p. Moreover, miR452-5p restoration led to obvious increase of HMGB3 enrichment in Ago2 group compared with that in $\mathrm{IgG}$ group (Fig. 5C).
Next, we probed the influence of miR-452-5p on HMGB3 expression. The data displayed that the mRNA and protein abundance of HMGB3 were reduced in the cells transfected with anti-miR-452-5p relative to anti-miR-NC group (Fig. 5D, E). In addition, mRNA and protein abundance of HMGB3 were elevated in PCa tissues and cells in comparison with their matched controls (Fig. 5F-I). Furthermore, miR-452-5p abundance was inversely correlated with HMGB3 level in PCa tissues $(r=-0.5766, p<0.0001)$ (Fig. 5J). Our findings suggested that miR-452-5p directly targeted HMGB3 in PCa cells.

\section{HMGB3 knockdown attenuated the promotive effects of miR-452-5p on growth and metastasis of PCa cells}

Although the interaction between miR-452-5p and HMGB3 was identified, the biological behaviors of $\mathrm{PCa}$ cells regulated by miR-452-5p and HMGB3 still need to be determined. Results implicated that the mRNA and protein 


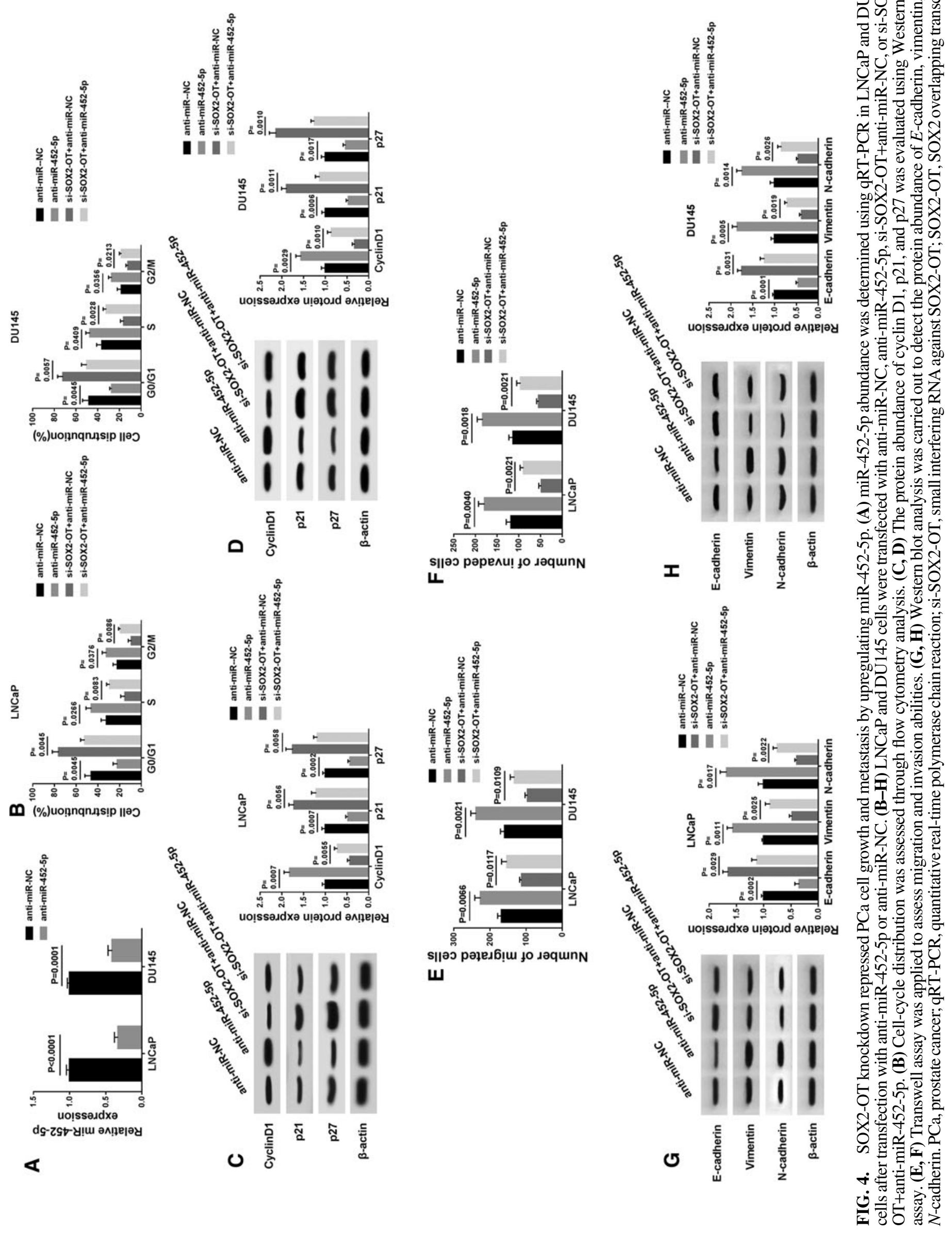



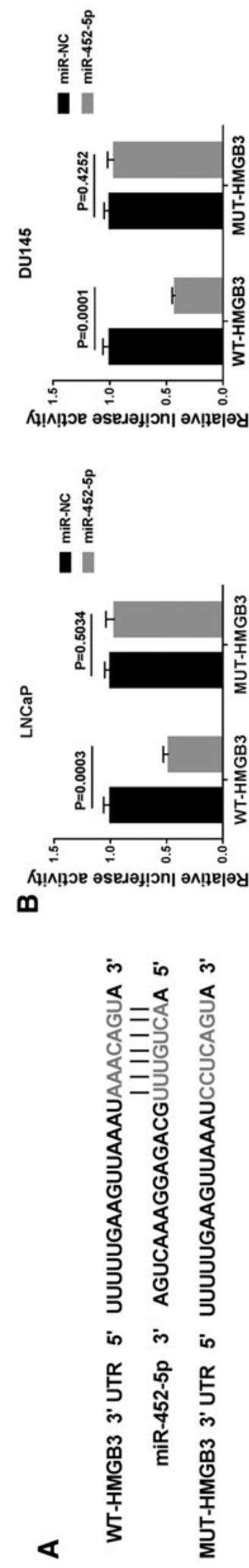

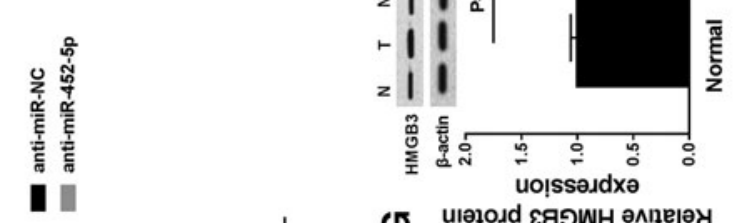

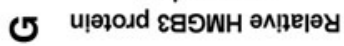

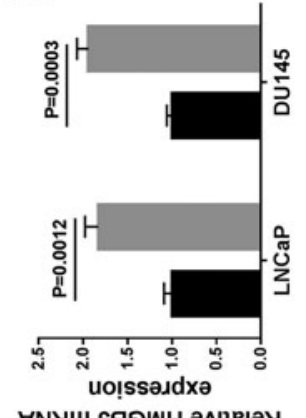

อ
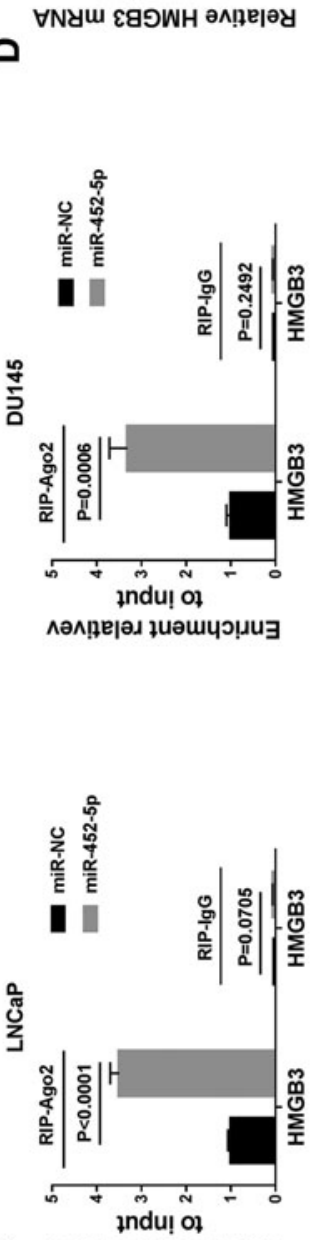

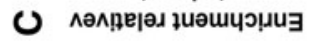

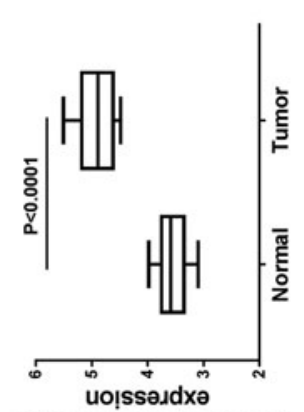

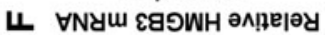

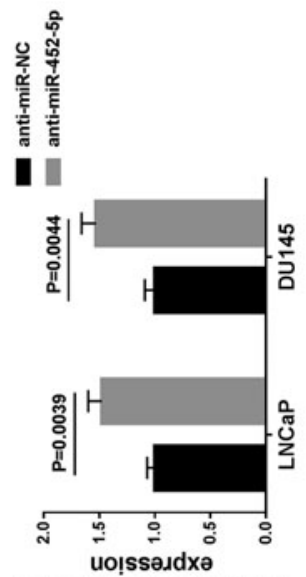

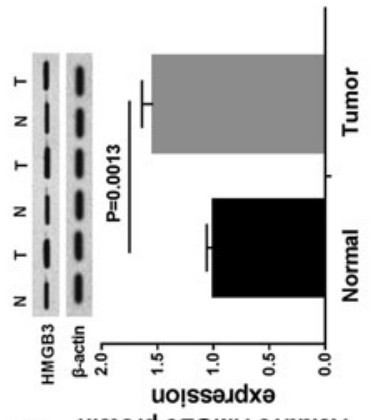

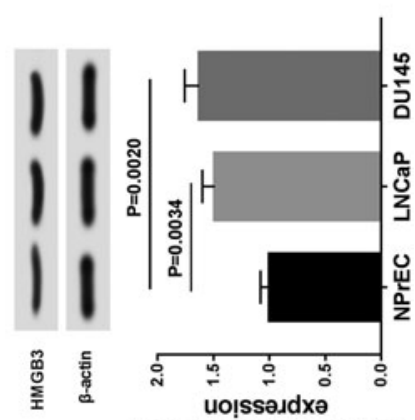

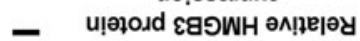

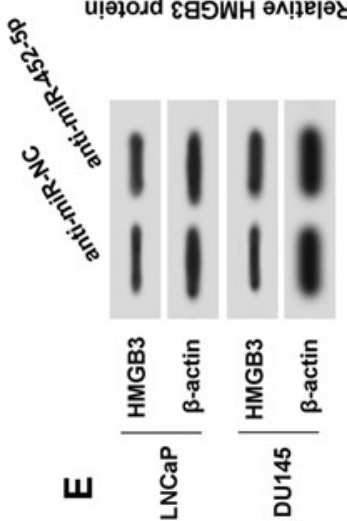

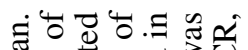

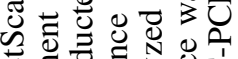

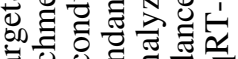

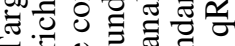

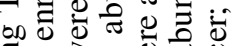

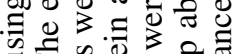

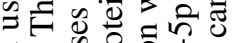

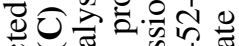

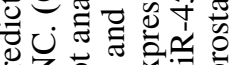

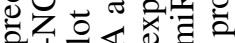
문

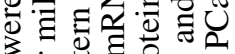
पे के 路巨可娄 서워 m的

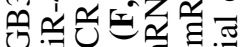

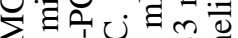

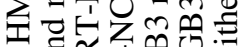
츰웡웡 ส

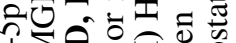
i 20 \&

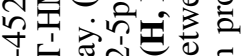

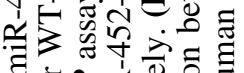

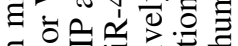

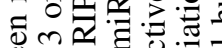
원

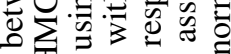

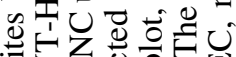
क

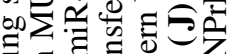

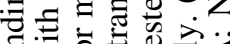

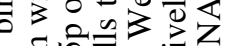
० ำ जु 눈 0 혀의을

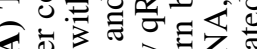

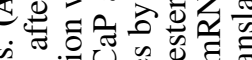
च تै s Uึ

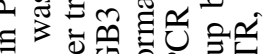
응 웡 कीज ब

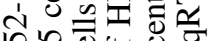

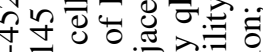

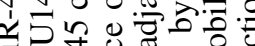

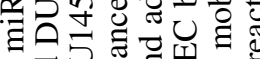
子?

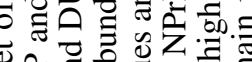

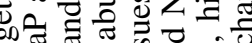
छั

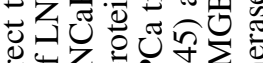

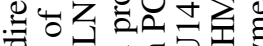

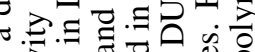

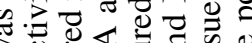
उ กิ Ð $\sum .0$ 品 ம่ ن들 
abundance of HMGB3 were decreased in si-HMGB3 in comparison with si-NC group (Fig. 6A, B). The results from flow cytometry analysis indicated that HMGB3 knockdown enhanced the ratio of cells in G0/G1 phase, whereas it reduced the ratio of cells in $\mathrm{S}$ and $\mathrm{G} 2 / \mathrm{M}$, and could reverse the anti-miR-452-5p-mediated reduction of G0/G1 phase cells and promotion of $\mathrm{S}$ and G2/M phase cells (Fig. 6C).

Meanwhile, Western blot assay demonstrated that interference of HMGB3 decreased the cyclin D1 expression and elevated the protein levels of p21 and p27, and weakened the effects of miR-452-5p knockdown by inhibiting cyclin D1 expression and enhancing p21 and p27 expression (Fig. 6D, E). Transwell assay manifested that inhibition of HMGB3 suppressed LNCaP and DU145 cell migration and invasion, and the promotive effects of miR-452-5p deficiency on migration and invasion were abated by downregulation of HMGB3 (Fig. 6F, G).

Furthermore, $E$-cadherin expression was elevated and the expression of vimentin and $N$-cadherin was declined after transfection of si-HMGB3, and cotransfection of si-HMGB3 and anti-miR-452-5p reversed the impact of miR-452-5p knockdown on inhibition of $E$-cadherin expression and promotion of vimentin and $\mathrm{N}$-cadherin expression (Fig. $6 \mathrm{H}$, I). To sum up, these results show that knockdown of miR452-5p promoted growth and metastasis of PCa cells through upregulation of HMGB3.

\section{SOX2-OT regulated HMGB3 expression by sponging miR-452-5p and modulated the Wnt/ $\beta$-catenin signaling pathway through affecting HMGB3 expression}

To probe whether SOX2-OT acted as a ceRNA to affect HMGB3 expression by sponging miR-452-5p, LNCaP and DU145 cells were transfected with si-NC, si-SOX2-OT, siSOX2-OT+anti-miR-NC, or si-SOX2-OT+anti-miR-452-5p to examine HMGB3 abundance. The results proved that transfection of si-SOX2-OT declined the mRNA and protein levels of HMGB3, whereas the effect was weakened by cotransfection of anti-miR-452-5p (Fig. 7A, B). The qRTPCR analysis confirmed the overexpression efficiency of SOX2-OT, which was demonstrated by the upregulation of SOX2-OT expression in pcDNA-SOX2-OT-transfected LNCaP and DU145 cells (Fig. 7C).

$\mathrm{Wnt} / \beta$-catenin signaling pathway is known to be activated in multiple cancers. ${ }^{22}$ Next, we explored the expression of proteins linked to the $\mathrm{Wnt} / \beta$-catenin signaling pathway. Western blot assay proved that accumulation of SOX2-OT enhanced the $\beta$-catenin and c-Myc protein levels, suggesting that $\mathrm{Wnt} / \beta$-catenin signaling pathway was activated by addition of SOX2-OT (Fig. 7D, 7E). Meanwhile, knockdown of HMGB3 alleviated the effect of SOX2-OT upregulation by activating $\mathrm{Wnt} / \beta$-catenin signaling pathway (Fig. 7D, E). Collectively, our results demonstrated that SOX2-OT/miR$452-5 \mathrm{p} / \mathrm{HMGB} 3 / \mathrm{Wnt} / \beta$-catenin axis played a crucial role in $\mathrm{PCa}$ progression.

\section{SOX2-OT interference inhibited PCa tumorigenesis in vivo}

We established a mice xenograft model in which the DU145 cells transfected with sh-SOX2-OT or sh-NC were injected subcutaneously into nude mice to assess the effect of SOX2-OT on $\mathrm{PCa}$ in vivo. As shown in Figures $8 \mathrm{~A}$ and $\mathrm{B}$, knockdown of SOX2-OT blocked tumor growth (decreased tumor volume and weight) in xenograft model. Next, the expression levels of SOX2-OT, miR-452-5p, and HMGB3 were examined in tumor tissues. As expected, qRT-PCR analysis showed that interference of SOX2-OT led to a significant reduction in SOX2-OT expression, whereas it led to an increase in miR-452-5p abundance in excised tumor masses (Fig. 8C, D). In addition, mRNA and protein expression of HMGB3 were prominently lower in tumor tissues from sh-SOX2-OT group than that in sh-NC group (Fig. 8E, F).

\section{Discussion}

$\mathrm{PCa}$ is considered to be one of the most frequent and malignant cancers in men. Although many treatment strategies have been developed for PCa, the treatments for the advanced patients are still challenging. ${ }^{23}$ A growing body of evidence has shown that IncRNAs are commonly dysregulated in a variety of cancers and may contribute to tumor growth and progression, including $\mathrm{PCa}{ }^{24,25}$ Hence, more work should be carried out to deeply elucidate the functional role and underlying mechanisms of lncRNAs in PCa.

LncRNA SOX2-OT, located on human chromosome $3 q 26.33$, has been shown to be overexpressed and closely linked to tumor progression in many human cancers. For example, Zhang and Li pointed out that SOX2-OT knockdown limited nasopharyngeal carcinoma cell proliferation and metastasis through the miR-146b-5p/HNRNPA2B1 pathway. ${ }^{26}$ Wang et al. pointed out that SOX2-OT level was enhanced in osteosarcoma tissues and cells, and acted as tumor promotor in modulating osteosarcoma cell proliferation and metastasis. ${ }^{27}$ Besides, Wo et al. proved that SOX2-OT expression was enhanced in $\mathrm{PCa}$ tissues and cells and its downregulation inhibited PCa cell growth and migration by regulating miR-369-3p/CFL2 axis. ${ }^{11}$ Nevertheless, the exact role and underlying mechanism of SOX2$\mathrm{OT}$ in $\mathrm{PCa}$ tumorigenesis remain largely unknown.

In this study we also observed that the SOX2-OT abundance was increased in PCa tissues and cells, and high SOX2-OT level was associated with poor overall survival. Moreover, we found that SOX2-OT interference arrested PCa cells in G0/G1 phase. Cyclin D1 is a critical oncogene that is required for the transition from the G1 to $S$ phase and for DNA replication, thereby contributing to cell proliferation. ${ }^{28}$ It has been reported that p21 and p27 function as CDK inhibitors to induce cell cycle arrest. ${ }^{19}$ In this study, results revealed that SOX2-OT knockdown repressed PCa cell growth by inhibiting cyclin D1 expression and promoting p21 and p27 abundance.

The EMT has been confirmed to be a critical process in cell metastasis of cancers. ${ }^{29,30}$ Our data showed that SOX2OT silence inhibited migration, invasion, and EMT by enhancing $E$-cadherin expression and reducing vimentin and $N$-cadherin expression. These results suggested that SOX2OT might serve as a tumor promoter in $\mathrm{PCa}$.

Recently, many researchers have demonstrated that lncRNAs exert their biological functional by sponging miRNAs to modulate gene expression. ${ }^{31,32}$ Based on this theory, starbase was applied to predict the potential targets of 


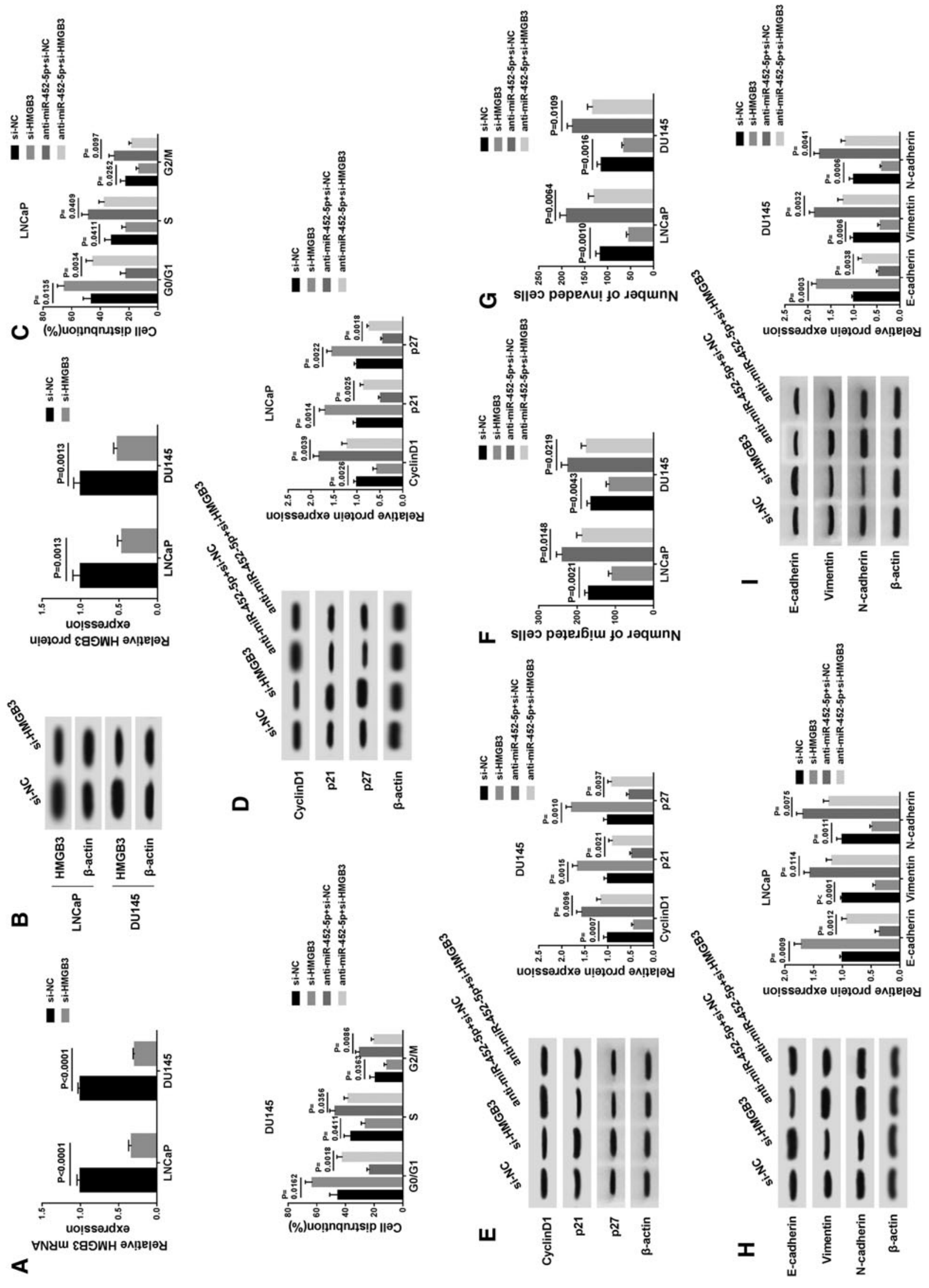

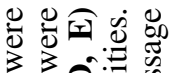

ne

गें

计呈

可: 물

元语.

ज琎

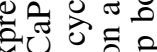

¿乙二

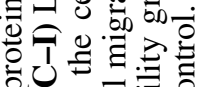

능

च

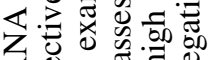

잉응

ठำ

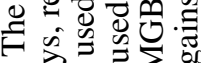

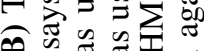

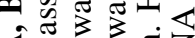

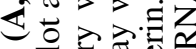

लํ월

कै हี छ च

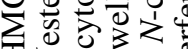

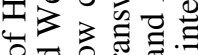

踏的

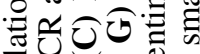

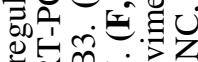

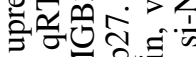

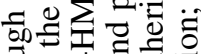

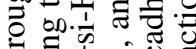

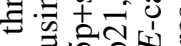

के जि

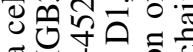

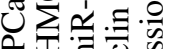

论市.

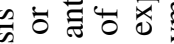

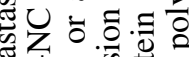

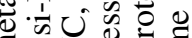

$\exists z=$

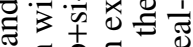

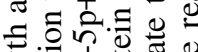

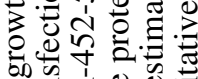

कo w w

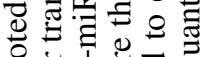

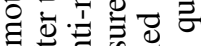
的范

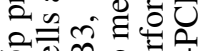

की

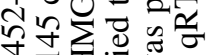

穴平了

站的希

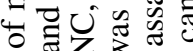

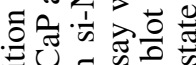

Z言告

画远远

.

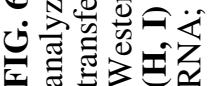




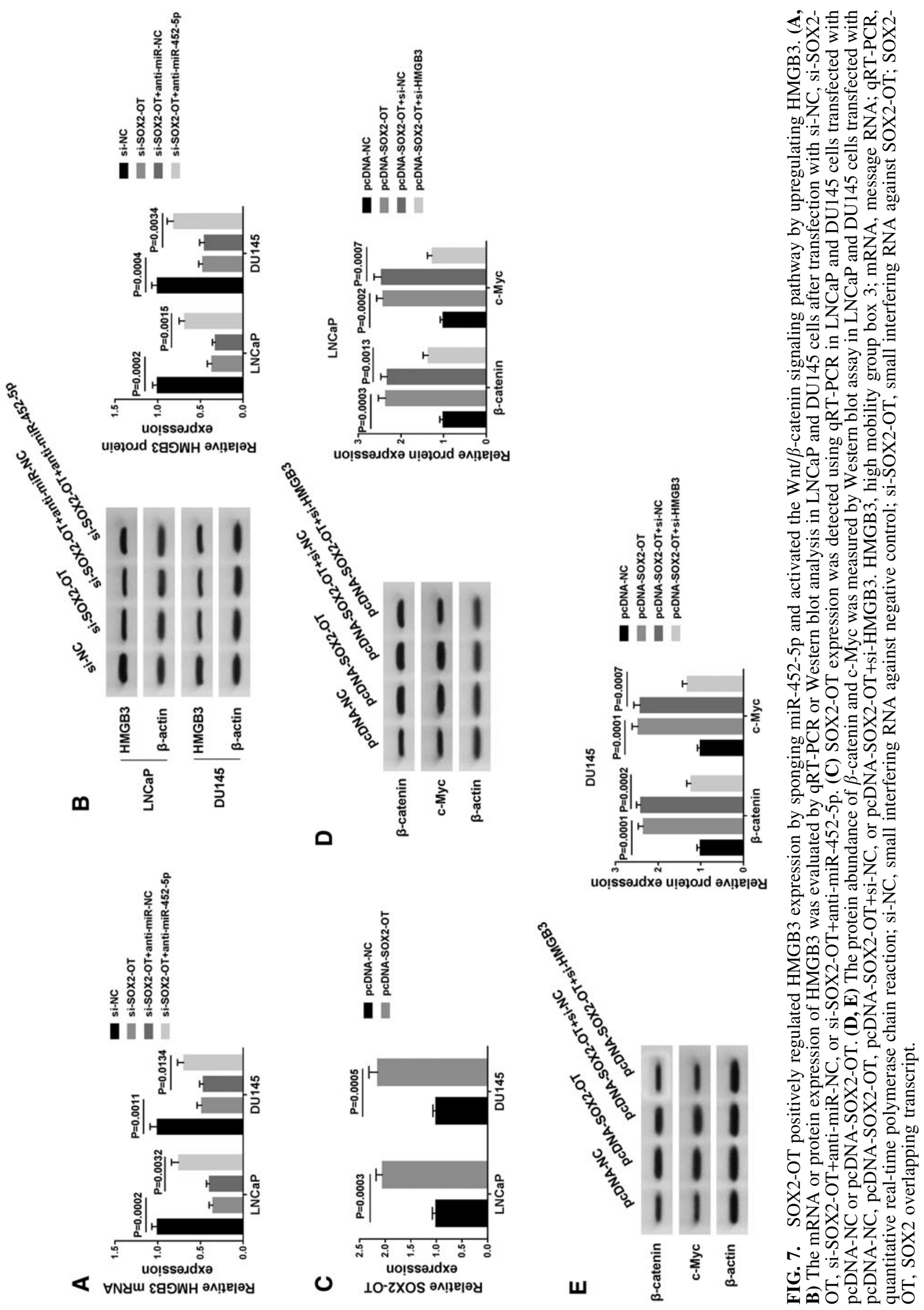


A
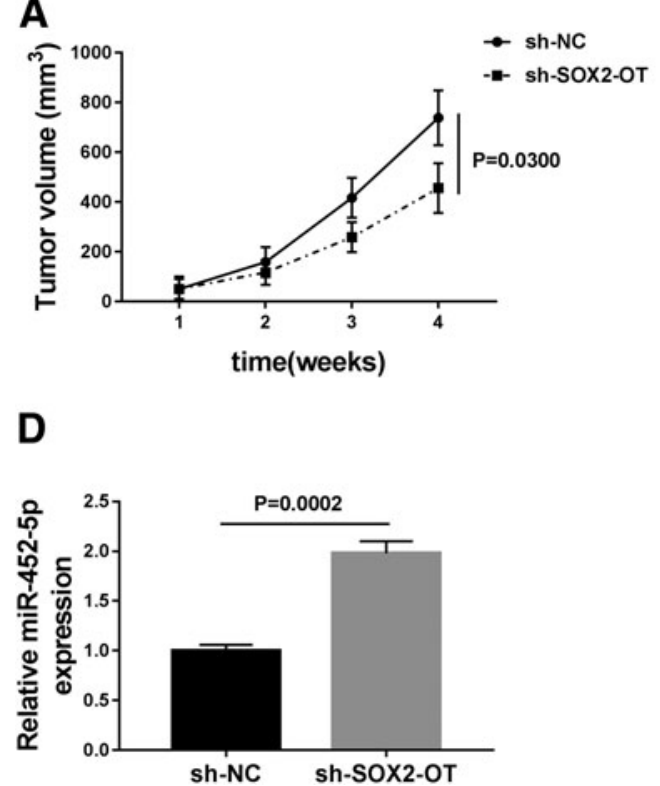

$B$
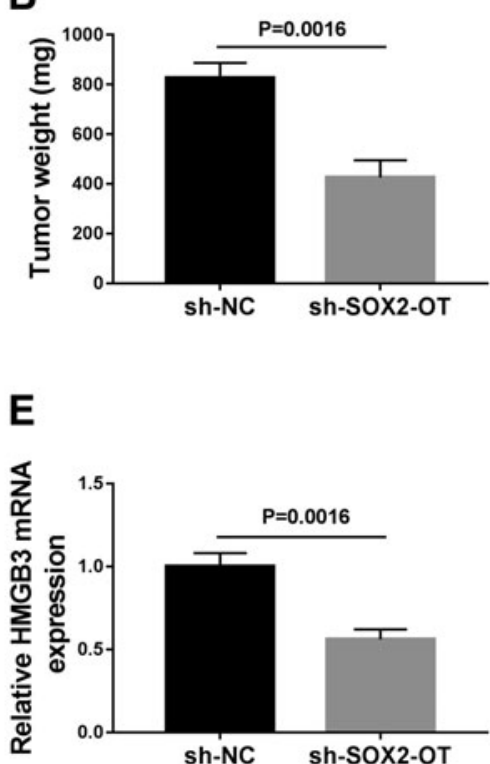
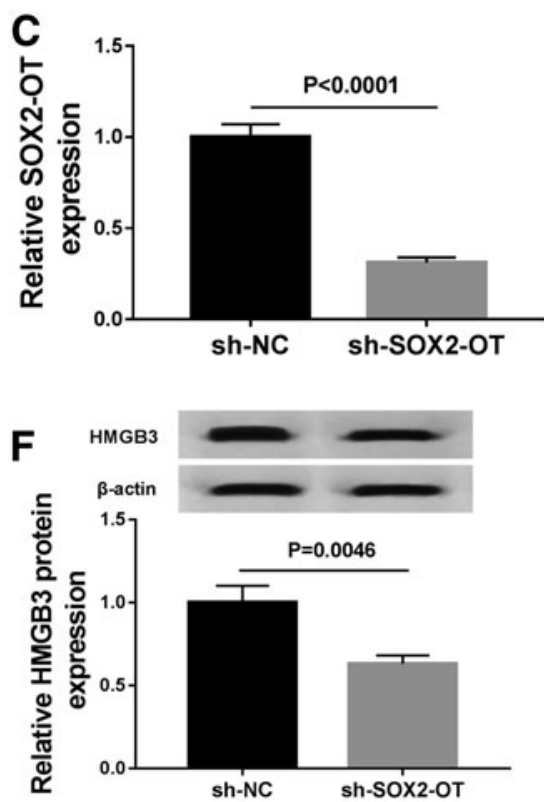

FIG. 8. Deficiency of SOX2-OT suppressed tumor growth through regulating miR-452-5p and HMGB3 expression in xenograft model. (A, B) DU145 cells transfected with sh-NC or sh-SOX2-OT were injected subcutaneously into nude mice and tumor volume and weight were detected. (C-E) The expression of SOX2-OT, miR-452-5p, and HMGB3 was measured through the qRT-PCR analysis in resected tumor tissues. (F) Western blot analysis was conducted to analyze the protein abundance of HMGB3 in resected tumor tissues. HMGB3, high mobility group box 3; qRT-PCR, quantitative real-time polymerase chain reaction; sh-NC, shRNA interference targeting negative control; SOX2-OT, SOX2 overlapping transcript.

SOX2-OT. The data showed that miR-452-5p might serve as a direct target of SOX2-OT, which was verified through dualluciferase reporter assay and RIP assay. MiR-452-5p has been identified to act as a tumor inhibitor or promoter, depending on the type of cancer. For instance, He et al. proved that miR-452-5p limited metastasis of lung cancer cells. ${ }^{33}$ Zheng et al. disclosed that miR-452-5p promoted hepatocellular carcinoma progression by targeting CDKN1B. ${ }^{34}$ Besides, some studies disclosed that miR-452-5p abundance was declined in PCa tissues and its restoration suppressed PCa cell migration and invasion. ${ }^{14,35,36}$

Consistent with these findings, we also demonstrated the miR-452-5p was lowly expressed in PCa tissues and cells. In addition, silencing miR-452-5p could reverse the suppressive impact of SOX2-OT downregulation on growth and metastasis of PCa cells. These findings indicated that SOX2OT knockdown inhibited the progression of PCa cells by upregulating miR-452-5p.

It is well known that miRNAs execute their biological role by modulating their target mRNAs. ${ }^{37} \mathrm{Next}$, we further probed the regulatory mechanism of miR-452-5p in the progression of PCa. Bioinformatics analysis showed that miR-452-5p had putative binding sites with HMGB3. Dualluciferase reporter and RIP assays manifested that miR452-5p directly targeted HMGB3. HMGB3 has been suggested to promote initiation and progression of multiple cancer. ${ }^{38,39}$ In addition, Yamada et al. revealed that HMGB3 mRNA expression was obviously enhanced in PCa tissues and its knockdown limited PCa cell growth, migration, and invasion. ${ }^{18}$

In this article, the data presented that HMGB3 mRNA and protein abundance were enhanced in PCa tissues and cells. Rescue experiments showed that the promotive impacts of miR-452-5p knockdown on growth and metastasis of $\mathrm{PCa}$ were attenuated by downregulation of HMGB3. All these data suggested that miR-452-5p exerted its biological function by modulating HMGB3 in PCa cells. Besides, we found that HMGB3 expression was negatively regulated by $\mathrm{miR}-452-5 \mathrm{p}$ and positively modulated by SOX2-OT. More importantly, SOX2-OT knockdown repressed glioma tumor growth in vivo by upregulating miR-452-5p and downregulating HMGB3. Collectively, these data suggested the SOX2-OT regulated progression of $\mathrm{PCa}$ by acting as a sponge for miR$452-5 \mathrm{p}$ to modulate HMGB3.

$\mathrm{Wnt} / \beta$-catenin signaling pathway can regulate multiple cell behaviors, including cell proliferation, differentiation, and invasion. $^{40}$ In addition, increasing evidence indicated that lncRNAs might participate in the development and progression of $\mathrm{PCa}$ by activating $\mathrm{Wnt} / \beta$-catenin signaling pathway. ${ }^{41,42}$ Thus, we further probed whether SOX2-OT contributed to the growth and metastasis of $\mathrm{PCa}$ by activating $\mathrm{Wnt} / \beta$-catenin pathway. In this research, the data showed that SOX2-OT overexpression could increase the protein abundance of $\beta$-catenin and $\mathrm{c}-\mathrm{Myc}$ in $\mathrm{PCa}$ cells, whereas silencing HMGB3 weakened the promotive effects. Therefore, these findings indicated that SOX2-OT deficiency suppressed the Wnt/ $\beta$-catenin signaling pathway by affecting HMGB3 expression.

\section{Conclusions}

In conclusion, our study showed that SOX2-OT knockdown inhibited PCa tumorigenesis and progression through 
miR-452-5p/HMGB3/Wnt/ $\beta$-catenin axis. These findings might provide new a novel mechanism for understanding $\mathrm{PCa}$ progression. Furthermore, SOX2-OT/miR-452-5p/ $\mathrm{HMGB} 3 / \mathrm{Wnt} / \beta$-catenin axis might serve as an innovative and prospective therapeutic target for $\mathrm{PCa}$.

\section{Authors' Contributions}

Conception and design: Y.S.; development of methodology: X.S.; acquisition of data: H.W.; analysis and interpretation of data: X.S., J.W.; writing, review, and revision of article: X.S. and Y.S.; all coauthors have reviewed and approved the article before submission.

\section{Disclosure Statement}

No competing financial interests exist.

\section{Funding Information}

No funding was received for this article.

\section{Supplementary Material}

Supplementary Table S1

Supplementary Table S2

\section{References}

1. Torre LA, Bray F, Siegel RL, et al. Global cancer statistics, 2012. CA Cancer J Clin 2015;65:2.

2. Ward JF, Sebo TJ, Blute ML, et al. Salvage surgery for radiorecurrent prostate cancer: Contemporary outcomes. J Urol 2005;173:4.

3. Wu XL, Zhang JW, Li BS, et al. The prognostic value of abnormally expressed lncRNAs in prostatic carcinoma: A systematic review and meta-analysis. Medicine (Baltimore) 2017;96:51.

4. Bartonicek N, Maag JL, Dinger ME. Long noncoding RNAs in cancer: Mechanisms of action and technological advancements. Mol Cancer 2016;15:1.

5. Majidinia M, Yousefi B. Long non-coding RNAs in cancer drug resistance development. DNA Repair 2016;45:25.

6. Li $\mathrm{Y}$, Luo $\mathrm{H}$, Xiao $\mathrm{N}$, et al. Long noncoding RNA SChLAP1 accelerates the proliferation and metastasis of prostate cancer via targeting miR-198 and promoting the MAPK1 pathway. Oncol Res 2018;26:1.

7. Wu M, Huang Y, Chen T, et al. LncRNA MEG3 inhibits the progression of prostate cancer by modulating miR-95p/QKI-5 axis. J Cell Mol Med 2019;23:1.

8. $\mathrm{Yu} \mathrm{H}, \mathrm{Xu} \mathrm{Q}$, Liu F, et al. Identification and validation of long noncoding RNA biomarkers in human non-small-cell lung carcinomas. J Thorac Oncol 2015;10:4.

9. Shahryari A, Rafiee MR, Fouani Y, et al. Two novel splice variants of SOX2OT, SOX2OT-S1, and SOX2OT-S2 are coupregulated with SOX2 and OCT4 in esophageal squamous cell carcinoma. Stem Cells 2014;32:1.

10. Askarian-Amiri ME, Seyfoddin V, Smart CE, et al. Emerging role of long non-coding RNA SOX2OT in SOX2 regulation in breast cancer. PLoS One 2014;9:7.

11. Wo Q, Zhang D, Hu L, et al. Long noncoding RNA SOX2OT facilitates prostate cancer cell proliferation and migration via miR-369-3p/CFL2 axis. Biochem Biophys Res Commun 2019;520:3.

12. Yoon J-H, Abdelmohsen K, Gorospe M (eds). Functional interactions among microRNAs and long noncoding RNAs. Semin Cell Dev Biol 2014:9.
13. Calin GA, Croce CM. MicroRNA signatures in human cancers. Nat Rev Cancer 2006;6:11.

14. Gao L, Zhang LJ, Li SH, et al. Role of miR-452-5p in the tumorigenesis of prostate cancer: A study based on the Cancer Genome Atl(TCGA), Gene Expression Omnibus (GEO), and bioinformatics analysis. Pathol Res Pract 2018;214:5.

15. Nemeth MJ, Curtis DJ, Kirby MR, et al. Hmgb3: An HMGbox family member expressed in primitive hematopoietic cells that inhibits myeloid and B-cell differentiation. Blood 2003;102:4.

16. Chen X, Zhao G, Wang F, et al. Upregulation of miR-513b inhibits cell proliferation, migration, and promotes apoptosis by targeting high mobility group-box 3 protein in gastric cancer. Tumour Biol 2014;35:11.

17. Xie X, Pan J, Han X, et al. Downregulation of microRNA$532-5 p$ promotes the proliferation and invasion of bladder cancer cells through promotion of HMGB3/Wnt/betacatenin signaling. Chem Biol Interact 2019;300.

18. Yamada $Y$, Nishikawa $R$, Kato $M$, et al. Regulation of HMGB3 by antitumor miR-205-5p inhibits cancer cell aggressiveness and is involved in prostate cancer pathogenesis. J Hum Genet 2018;63:2.

19. Coqueret O. New roles for p21 and p27 cell-cycle inhibitors: A function for each cell compartment? Trends Cell Biol 2003;13:2.

20. Baldin V, Lukas J, Marcote MJ, et al. Cyclin D1 is a nuclear protein required for cell cycle progression in G1. Genes Dev;7:5.

21. Kudo-Saito C, Shirako H, Takeuchi T, et al. Cancer metastasis is accelerated through immunosuppression during Snail-induced EMT of cancer cells. Cancer Cell 2009;15:3.

22. Hoffmeyer K, Raggioli A, Rudloff $\mathrm{S}$, et al. Wnt/ $\beta$-catenin signaling regulates telomerase in stem cells and cancer cells. Science 2012;336:6088.

23. Petrylak DP, Tangen CM, Hussain MHA, et al. Docetaxel and estramustine compared with mitoxantrone and prednisone for advanced refractory prostate cancer. N Engl J Med 2004;351:1513.

24. Gutschner T, Diederichs S. The hallmarks of cancer: A long non-coding RNA point of view. RNA Biol 2012;9:6.

25. Peng WX, Koirala P, Mo YY. LncRNA-mediated regulation of cell signaling in cancer. Oncogene 2017;36:41.

26. Zhang E, Li X. LncRNA SOX2-OT regulates proliferation and metastasis of nasopharyngeal carcinoma cells through miR-146b-5p/HNRNPA2B1 pathway. J Cell Biochem 2019;120:10.

27. Wang Z, Tan M, Chen G, et al. LncRNA SOX2-OT is a novel prognostic biomarker for osteosarcoma patients and regulates osteosarcoma cells proliferation and motility through modulating SOX2. IUBMB Life 2017;69:11.

28. Stacey DW. Cyclin D1 serves as a cell cycle regulatory switch in actively proliferating cells. Curr Opin Cell Biol 2003; 15:2.

29. Guarino M, Rubino B, Ballabio G. The role of epithelialmesenchymal transition in cancer pathology. Pathology 2007;39:3.

30. Xu J, Lamouille S, Derynck R. TGF- $\beta$-induced epithelial to mesenchymal transition. Cell Res 2009;19:2.

31. Sanchez-Mejias A, Tay Y. Competing endogenous RNA networks: Tying the essential knots for cancer biology and therapeutics. J Hematol Oncol 2015;8:30.

32. Du Z, Sun T, Hacisuleyman E, et al. Integrative analyses reveal a long noncoding RNA-mediated sponge regulatory network in prostate cancer. Nat Commun 2016;7:10982. 
33. He Z, Xia Y, Pan C, et al. Up-regulation of MiR-452 inhibits metastasis of non-small cell lung cancer by regulating BMI1. Cell Physiol Biochem 2015;37:1.

34. Zheng Q, Sheng Q, Jiang C, et al. MicroRNA-452 promotes tumorigenesis in hepatocellular carcinoma by targeting cyclin-dependent kinase inhibitor 1B. Mol Cell Biochem 2014;389:1.

35. Goto Y, Kojima S, Kurozumi A, et al. Regulation of E3 ubiquitin ligase-1 (WWP1) by microRNA-452 inhibits cancer cell migration and invasion in prostate cancer. $\mathrm{Br} \mathbf{J}$ Cancer 2016;114:10.

36. Kristensen H, Haldrup C, Strand S, et al. Hypermethylation of the GABRE $\sim \operatorname{miR}-452 \sim$ miR-224 promoter in prostate cancer predicts biochemical recurrence after radical prostatectomy. Clin Cancer Res 2014;20:8.

37. Bartel DP. MicroRNAs: Target recognition and regulatory functions. Cell 2009;136:2.
38. Zhang Z, Chang Y, Zhang J, et al. HMGB3 promotes growth and migration in colorectal cancer by regulating WNT/beta-catenin pathway. PLoS One 2017;12:7.

39. Guo S, Wang Y, Gao Y, et al. Knockdown of high mobility group-box 3 (HMGB3) expression inhibits proliferation, reduces migration, and affects chemosensitivity in gastric cancer cells. Med Sci Monit 2016;22:3951.

40. Fodde R, Brabletz T. Wnt $/ \beta$-catenin signaling in cancer stemness and malignant behavior. Curr Opin Cell Biol 2007;19:2.

41. Li JB, Liu F, Zhang BP, et al. LncRNA625 modulates prostate cancer cells proliferation and apoptosis through regulating the $\mathrm{Wnt} /$ beta-catenin pathway by targeting miR432. Eur Rev Med Pharmacol Sci 2017;21:11.

42. Song J, Wu X, Ma R, et al. Long noncoding RNA SNHG12 promotes cell proliferation and activates Wnt/beta-catenin signaling in prostate cancer through sponging microRNA195. J Cell Biochem 2019;120:8. 